\title{
The Changes In Calendars In The Ancient World As A Tool To Teach The Development Of Astronomy
}

\author{
Ariel Cohen, The Hebrew University of Jerusalem, Israel
}

\begin{abstract}
When teaching an introductory science survey course to college students learning astronomy at the Hebrew University of Jerusalem, Israel, we have devoted four hours to teaching the history of astronomy as a fruitful strategy to introduce important concepts surrounding the development of general scientific knowledge throughout history. In order to illustrate the impact of improved accuracy of astronomical measurements, we propose using the example the development of the calendars and, in particular, the widespread Hebrew calendars used throughout the adjacent Millennia of B.C. and A.C. The changes in the several determinations of the Hebrew calendar are demonstrated based on Babylonian and Jewish documents as well as works by al-Khwarizmi from the 9th century AD, found in the Khuda Bakhsh Oriental Library, in Patna India. Our experience suggests that the teaching of calendar development and evolutions demonstrates the interconnectedness between scientific endeavors and social-religious traditions.
\end{abstract}

Keywords: Teaching of Astronomy; Archeoastronomy; Calendars and Time Keeping; Al-Khwarizmi; Biblical Chronology; Astronomy and Religion; Ancient Cultures and Astronomy; Dating

\section{INTRODUCTION}

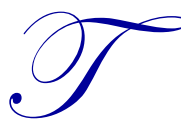

he teaching of Astronomy provides a most valuable tool to grant to the students the knowledge and understanding of the development of science in general throughout the history of mankind. In particular, it offers an understanding of the role of religion in the development of science: Had there always been a confrontation between religious beliefs attempting to interfere with new discoveries which may contradict them?

It is generally recognized that the brilliant discoveries of Galileo were considered by the Catholic Church as a strong case for convicting him of heresy, and thus brought to a temporary halt the progress of science. But it is not a common knowledge that such a confrontation between religion and science had been practically none existing in many cases in ancient times. On the opposite, it can be shown, as we shall demonstrate below, that several basic religious laws were derived from the contemporary scientific, in general, and astronomical, in particular, findings. In many generations, astronomy and Religion or cultural laws and habits had been developed step by step together.

One of the best platforms to learn and understand such developments is the teaching of astronomy which provides not only the present picture of the sky and its corresponding physical laws, but also the historical developments leading to our present insight and wisdom.

We chose as an example the changing visualization of the formation of the Earth and the Universe. It is clear that in all ancient cultures it had been recognized that there could not be any document other than astronomical descriptions including celestial coordinates of various elements in the sky that could be related to the creation of the universe. In the first millennium B.C. and the first centuries A.C. the scientific knowledge about the age of the world was very vague, but in several cultures, it was accepted that the age of the present Earth did not exceed a few thousand years. As an illustration we can refer to the Kaly-Yuga scale of the age of the present cycle of the world of Hinduism: 
According to the Hindu tradition the present cycle started at 3102 B.C. This was the year that the astronomer Aryabhatta calculated in 476 A.C. to be the starting point of the Kaly-Yuga because in that year there had been an extremely rare planetary alignment - all 7 planets including the moon and the sun had been in the same celestial longitude (Burgess, 1860). This unique arrangement of the planets was believed to have been required for describing the unique cultural event in Hinduism when Krishna left the Earth, returned to his heavenly adobe and the epoch of their new era of 4,320,000 years began (See Figure 1).

Figure 1. The Hindu cycle of 4,320,000 sidereal years after which all planets as well as the lunar node and the lunar apogee complete each an integer number of cycles and thus return to the same common celestial longitudes.

\section{Mean Motions of the Planets:}

For Example : $29 ; 31,50.0 .7=29.5305588$ close to the Islamic Month

\begin{tabular}{|c|c|c|c|c|}
\hline Planet. & $\begin{array}{c}\text { Number of } \\
\text { revolutions in } \\
4,320,000 \text { yoars. }\end{array}$ & $\left|\begin{array}{c}\text { Number of } \\
\text { revolutions in } \\
1,080,000 \text { years. }\end{array}\right|$ & $\begin{array}{l}\text { Length of a revolution } \\
\text { in mean wolar time. }\end{array}$ & Mean daily motion. \\
\hline $\begin{array}{l}\text { Sun, } \\
\text { Mercury, } \\
\text { Venus, } \\
\text { Mars, } \\
\text { Jupiter, } \\
\text { Saturn, } \\
\text { Moon: } \\
\text { sider. rev. } \\
\text { synod. rev. } \\
\text { rev. of apsis, } \\
\text { “ “ node, }\end{array}$ & $\begin{array}{r}4,320,000 \\
17,937,060 \\
7,022,376 \\
2,296,832 \\
364,220 \\
146,568 \\
\\
57,753,336 \\
53,433,336 \\
488,203 \\
232,238\end{array}$ & $\begin{array}{r}1,080,000 \\
4,484,265 \\
1,755,594 \\
574,208 \\
91,055 \\
36,642 \\
\\
14,438,334 \\
13,358,334 \\
122,050 \frac{8}{4} \\
58,059 \frac{1}{3}\end{array}$ & \begin{tabular}{|rrrr}
$\mathrm{d}$ & $\mathrm{n}$ & $\mathbf{v}$ & $\mathrm{p}$ \\
365 & 15 & 31 & 3.14 \\
87 & 58 & 10 & 5.57 \\
224 & 41 & 54 & 5.06 \\
686 & 59 & 50 & 5.87 \\
4,332 & 19 & 14 & 2.09 \\
10,765 & 46 & 23 & 0.41 \\
& & & \\
27 & 19 & 18 & 0.16 \\
29 & 31 & 50 & 0.70 \\
3,232 & 5 & 37 & 1.36 \\
6,794 & 23 & 59 & 2.35
\end{tabular} & $\begin{array}{rrrrr} & 1 & \prime \prime & \cdots & \cdots . . \\
& 59 & 8 & 10 & 10.4 \\
4 & 5 & 32 & 20 & 41.9 \\
1 & 36 & 7 & 43 & 37.3 \\
31 & 26 & 28 & 11.1 \\
& 4 & 59 & 8 & 48.6 \\
& 2 & 0 & 22 & 53.4 \\
& & & & \\
13 & 10 & 34 & 52 & 3.8 \\
12 & 11 & 26 & 41 & 53.4 \\
6 & 40 & 58 & 42.5 \\
3 & 10 & 44 & 43.2\end{array}$ \\
\hline
\end{tabular}

The Figure can be found on page 161 in Burgess, E. (1860). Surya-Siddhnta, a Text-Book of Hindu Astronomy: https://archive.org/stream/jstor592174/592174\#page/n21/mode/2up

We shall now deal with the epochs of the world as had been determined based on the Old Testament.

\section{The Determination of the Age of the Universe in Astrophysics and in the Jewish Tradition}

A group of students from Jerusalem was visiting Moscow during the autumn of 2017. When brought by their guide to the main library in Moscow, they saw at the entrance a document in which the main Russian historical events of the second Millennium A.C. (Anno Christi) were displayed (Fig. 2). 
Figure 2. A list of major historical events in Russia between the years 1534 and 1727 A.C. as documented in a library in Moscow.

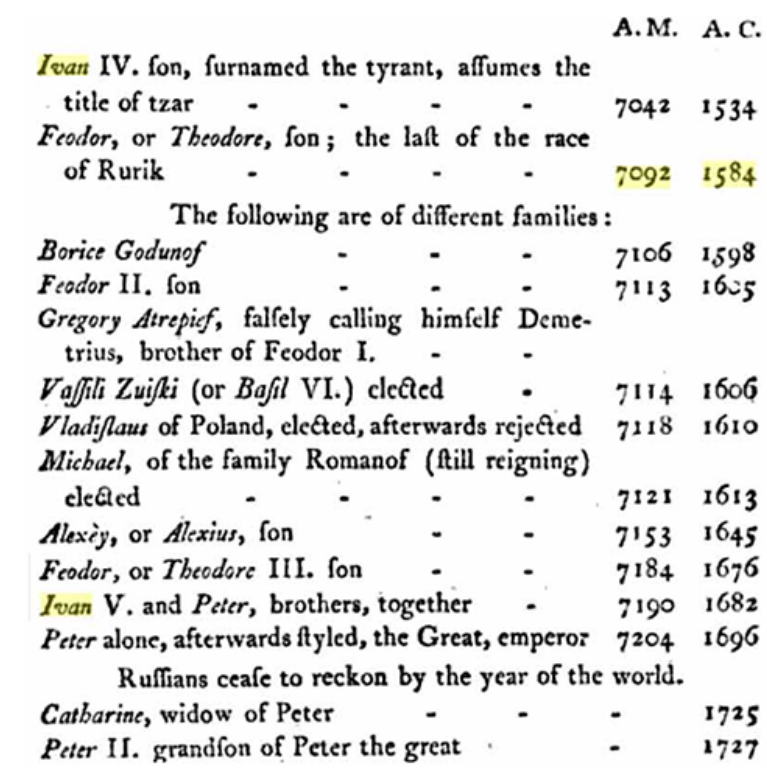

The Figure can be found on page 439 in Jean-Henri Castéra, The life of Catharine II, Empress of Russia Vol1 https://books.google.co.il/books?id=Xd0KAAAAIAAJ.

From Figure 2 we can learn, for example, that Ivan the Tyrant assumed the title of a tzar in the year 1534 A.C. The guide of the group added that in Russia up until the year 1700 A.C., all official documents included also the corresponding year "from creation" which was in our example the year 7042 A.M. (Anno Mundi). When the members of the group requested an explanation about the foundations of this "Anno Mundi" scale, the guide was flabbergasted: "You have just arrived from the land of the Bible and I would assume that you know that the year from creation is the accumulated sum of the ages of the Patriarchs from Adam to Abraham and the following biblical chronology up until the destruction of Solomon's Temple, as detailed in the Bible. Since we have historical records according to which the destruction took place in the year 586 B.C., we can now go back and determine the "year of creation" (YCR).

The reply he received from the surprised group of visitors was very enigmatic to him: "We have just celebrated the new year ("Rosh Hashanah") of the year 5778 A.M. that had been determined using a similar method to the one you have just described".

The two sides were unable to resolve the enigma because they were unaware of the fact that there are different years of creation applying to more than one version of the bible.

The two major versions are known as the "Septuagint" version which is the Old Testament believed to be the authentic version by the Greek Orthodox Church, mainly in Russia as well as in Armenia, and the "Masoretic" version used currently by practically all other Christians and by the Jews all over the world. One of the major differences between the two versions is the chronology as can be seen in Table 1 where, for example, the ages of several of the Patriarchs when they begot their following generations differ by 100 years each. 
Table 1. The ages of the Patriarchs when they begot their following generation. Therefore, Noah gave birth to his sun Shem 2164 years after creation.

\begin{tabular}{l|c|c}
\hline & Septuagint & \multicolumn{2}{c}{ Masoretic } \\
\hline Patriarch & Begot & Begot \\
\hline Adam & 230 & 130 \\
\hline Seth & 205 & 105 \\
\hline Enosh & 190 & 90 \\
\hline Kenan & 170 & 70 \\
\hline Mahalalel & 165 & 65 \\
\hline Jared & 162 & 162 \\
\hline Enoch & 165 & 65 \\
\hline Methuselah & 167 & 187 \\
\hline Lamech & 188 & 182 \\
\hline Noah & 502 & 502 \\
\hline
\end{tabular}

* The Vaticanus Manuscript.

Table 1 and other chronological differences lead to the fact that the year 5778 A.M. in Israel, is the year 7526 A.M. for the Greek Orthodox Church.

The question raised here is what were the reasons for using first one of the two versions of the chronologies of the Old Testament and modify it to the other version? - This question is inevitable after a comparison between the ages of the Patriarchs in the two versions - there is no doubt that one list of ages was derived from the other.

Below we show that the two chronologies which may be both, new versions of another original script, are a reflection of the advances in the accuracy of astronomical measurements in ancient times. We show that the ancient sages had visualized the positions of the planets when they were formed and by knowing the daily motions of each star they calculated backwards and looked for the year in which the stars' celestial longitudes were in agreement with their predetermined conception.

Such an approach reveals that there are profound similarities between this ancient scientific approach and the modern astrophysical methodology used in the present determination of the age of the universe: In astrophysics it is assumed that at first there was the big bang followed by the expansion of the universe. If one can now determine the "Hubble's Constant" with a high degree of accuracy, a backward extrapolation of the varying distance between stars can lead to the answer as to when they all started from a practically zero distance. But, with the introduction of constantly improving sophisticated methods enabling more accurate determinations of the value of Hubble's constant including the findings of its acceleration the age of the universe had been changed from 12 to 13.7 billion years, a modification that took place within the past 30 years. In Science, the change of the value of a "Physical Constant" has never been regarded as a failure of the older system but rather as an important step forward due to the progress of science. Indeed the ability to calculate the age of the universe with the most sophisticated methods granted the Nobel prize in Physics in 2011 to Adam Riess, Brian Schmidt and Saul Perlmutter.

We show that the scientific progress is also the reason that made it necessary to change the Anno Mundi scale in the ancient world - a backward extrapolation of the planetary positions, leading to the presumed visualization of their positions when they were formed, using more accurate astronomical input, led to a different "YCR" than the one that was found using older data. As a consequence, a new version of the biblical chronology had been designed.

But how do we know what was the visualization of the positions of the stars in the sky in the day of creation?

We found the answer hidden in a rare manuscript written by one of the best known astronomers of the first Millennium A.C., Muhammad ibn Musa al-Khwarizmi (Kennedy, 1964). 


\section{THE PLANETS ON THE DAY OF CREATION}

Al-Khwarizmi (780-866 AD), the founder of Algebra, had also several contributions in the fields of Geography and Astronomy. His reputation went far beyond the geographical boundaries of his native city of Chiva in Uzbekistan where he flourished early in the $9^{\text {th }}$ century A.C. and he was invited by Calif al-Mamun in Baghdad to become the chief astronomer in his court. al-Khwarizmi accepted the offer and started to partially devote himself to the education of astronomy. He knew that the progress of science depends on the existence of a library which contains all the past documents summarizing his contemporary scientific state of the art. In order to achieve that goal he accumulated all documents that he could find. One of such documents was an astronomical Table the most important content of which was so well hidden in the text that even he himself only reported the existence of the Table without discussing its input.

But, al-Khwarizmi felt that it was necessary to present its astronomical information which, as will be shown below, is a treasure to the understanding of the Biblical chronology. It is the only document in existence that provides such important clues to our understanding the chronology:

He did so in one of his important treatises in astronomy which he devoted to the new permanent luni-solar Jewish calendar's regulations (Kennedy, 1964). In that work he incorporated this old Astronomical Table (Table 2), which included the celestial positions of the planets on the first days of Adam (See part of the original manuscript in Figure $3)$.

Figure 3. The first two positions as appeared in the unique manuscript copy found on folios $115 \mathrm{v}-117 \mathrm{r}$ of MS. Bankipore Arabic 2468 in the Khuda Bakhsh Oriental Public Library, Patna, India: "The mean sun on the first of the days of Adam, on a Friday: 526 (in Arabic letters), the mean moon: 5 26."

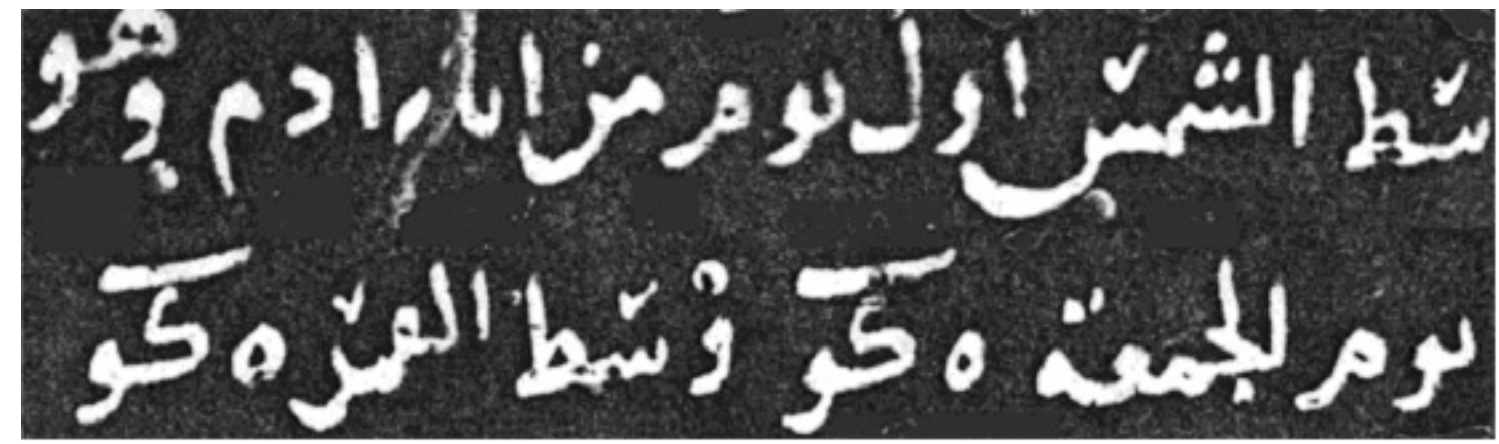

For the first time, in the middle of the $20^{\text {th }}$ century A.D., astronomers were exposed to a table that was practically buried in the basement of a library in Patna India since the $9^{\text {th }}$ century A.C. a table that presents the celestial longitudes of all planets in 3 major epochs: 1. as mentioned above, the Biblical day of creation, 2. - the beginning of the era of Solomon's Temple, and 3. - the epoch, Se, of the Seleucid era, named by al-Khwarizmi as the "Two Horned" epoch. For us it was a pearl that had to be decoded. In the original work there is no reference to the origin of the table but as we discuss below it contains data that can be attributed only to pre-Talmudic era, namely, before the $3^{\text {rd }}$ century A.C., an era in which it was believed that the world was created in the spring. The table, has thus three columns of data and in our present work, we shall analyze the set of positions of all planets in the first column and just concentrate on the coordinates assigned to the mean sun and the mean moon at the epoch of Solomon's Temple era and briefly discuss those at the epoch, Se, of the "Two-Horned" era. 
Table 2. The positions of the planets as reported by Kennedy, 1964 based on al-Khwarizmi's treatise. For the last value of the second column 5 14, see Fig. 4.

\begin{tabular}{|c|c|c|c|}
\hline 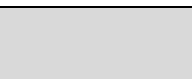 & $\begin{array}{c}\text { The first of the days of Adam. } \\
\text { A Friday }\end{array}$ & The building of the Temple & $\begin{array}{c}\text { The beginning of the years of } \\
\text { the Two Horned }\end{array}$ \\
\hline Mean sun & 526 & 526 & $613 ; 31,18$ \\
\hline Mean moon & 526 & 526 & $46 ; 45,49$ \\
\hline Lunar apogee & 15 & $926 ; 40,16$ & $726 ; 17.19$ \\
\hline Saturn & 815 & $1022 ; 9,1$ & $88 ; 24,6$ \\
\hline Jupiter & 65 & $37 ; 42,34$ & $312 ; 52,18,13$ \\
\hline Mars & 16 & $115 ; 26,21$ & $812 ; 14,46$ \\
\hline Venus & 425 & $712 ; 11,47$ & $21 ; 223$ \\
\hline Mercury & $\begin{array}{c}\text { The coordinate is missing in the } \\
\text { original work }\end{array}$ & $113 ; 19,19$ & $710 ; 118$ \\
\hline Lunar node & $514^{*}$ & $426 ; 34,11$ & $423 ; 41,27$ \\
\hline
\end{tabular}

Figure 4. "The node 5 14". As can be seen, the alphanumeric values of the handwritten letters should be inferred to denote the $164^{\text {th }}$ degree, and not the $14^{\text {th }}$ degree as was wrongly inferred by Kennedy, 1964 . The value of 164 is the only meaningful value for the lunar node as explained below at the end of section 3. This can also be found in the unique manuscript copy found on folios $115 \mathrm{v}-117 \mathrm{r}$ of MS. Bankipore Arabic 2468 in the Khuda Bakhsh Oriental Public Library, Patna, India.

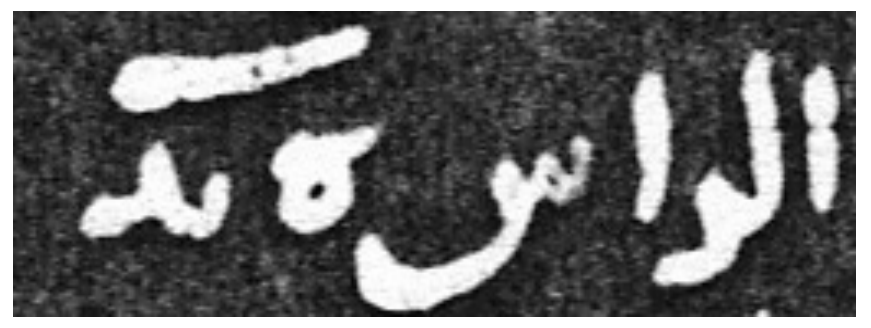

In his 1964 article Kennedy discussed the basic properties of the positions of the planets and made the following remarks which have remained unchallenged except for by us:

"All entries are mean rather than true positions and those for the inferior planets are anomalies rather than mean longitudes."

He then added that

"al-Biruni remarks that some of the Jews claim that the 'autumnal equinox' took place at the end of the third hour of the Day of Wednesday, the $5^{\text {th }}$ of Tishri after Creation'. Our text has the mean sun at epoch, four degrees before the autumnal equinox, which is consistent with the above. But beyond this the numbers for the first two sets of positions seem to make little sense." (See Fig. 5) 
Figure 5. The visualization of the celestial longitudes of the planets as suggested by al-Khwarizmi's Table, and as claimed by Kennedy, 1964, to be meaningless. This Figure was created by the author.

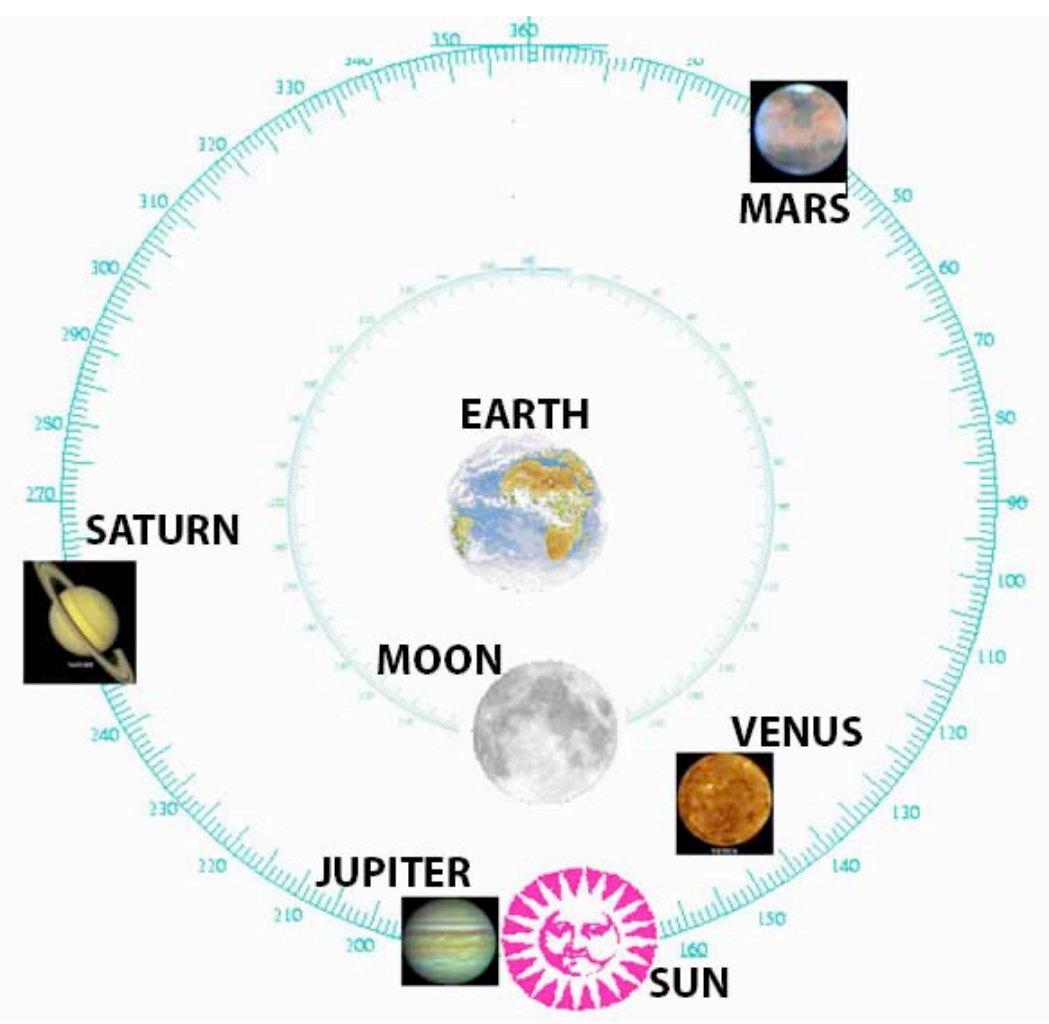

But, as we show below, Kennedy was misled by a misrepresentation of the data for "the first of the days of Adam", and, consequently, was inaccurate in several of his major statements:

A. The numbers of the first set have a significant meaning which reveals the basic understanding of the solar system and the first new moon in the Jewish day of creation of the stars and planets. As we show the Table above had been modified from a different original Table from which we can learn how the sky was visualized by the ancient astronomers who composed the original Table in the last centuries B.C. or the first centuries A.D.

B. The position of the inferior planet Venus in the first column is the true position and not the anomaly (the position of Mercury is missing from the original text). - See Appendix 1.

In fact, when the longitudes in the set of positions of the sun, the moon and the planets given for "the first of the days of Adam", are rearranged, their assigned values are ingenious: They astonishingly form a unique order and as we show below the celestial coordinates of the planets, and, in particular that of Venus are resulting from advanced astronomical computations.

\section{THE HIDDEN POSITIONS OF THE "OUTER" PLANETS IN THE DAY OF THEIR CREATION}

In our attempts to understand what had been the unseen message which motivated the writers of the original Table to construct the first set of positions as they are, we tried to determine from the set of positions given for the time of the new moon close to the autumn ("Adam's new moon"), what would be the corresponding assumed positions in the preceding spring. 
For that we calculated the positions for six lunar months earlier when the sun, the moon and the other planets were believed by several ancient Jewish sages to have been created.

At first, when we based our calculations on the motions of the planets, the results at the beginning of spring made also little sense, as stated by Kennedy. But, when we found out, as will be elaborated below, that the YCR in the original Table made for the spring creation, is 3 years (37 lunar months) after the new believed to be the YCR, we assumed that the astronomical positions of Saturn and Jupiter were interchanged in the handwritten Table as inserted by alKhwarizmi into his manuscript. Just by interchanging the 2 positions the modified Table offered a most interesting result:

First, we got the following corrected set of coordinates for the sun and the planets (Table 3):

Table 3. The corrected positions of the planets. (See Fig 6)

\begin{tabular}{l|c|c}
\hline & The first of the Days of Adam & In Degrees \\
\hline Saturn & $6^{\mathrm{s}} 5^{0}=6 \times 30+5$ & $185^{\text {th }}$ degree \\
\hline Jupiter & $8^{\mathrm{s}} 15^{0}$ & $255^{\text {th }}$ degree \\
\hline Mars & $1^{\mathrm{s}} 6^{0}$ & $36^{\text {th }}$ degree \\
\hline Mean sun & $5^{\mathrm{s}} 26^{0}$ & $176^{\text {th }}$ degree \\
\hline Venus & $4^{\mathrm{s}} 25^{0}$ & $145^{\text {th }}$ degree \\
\hline
\end{tabular}

Figure 6. The visualization of the modified celestial longitudes of the planets. This Figure in which Jupiter's and Saturn's original al-Khwarizmi's celestial longitudes were interchanged by the author, was create by us.

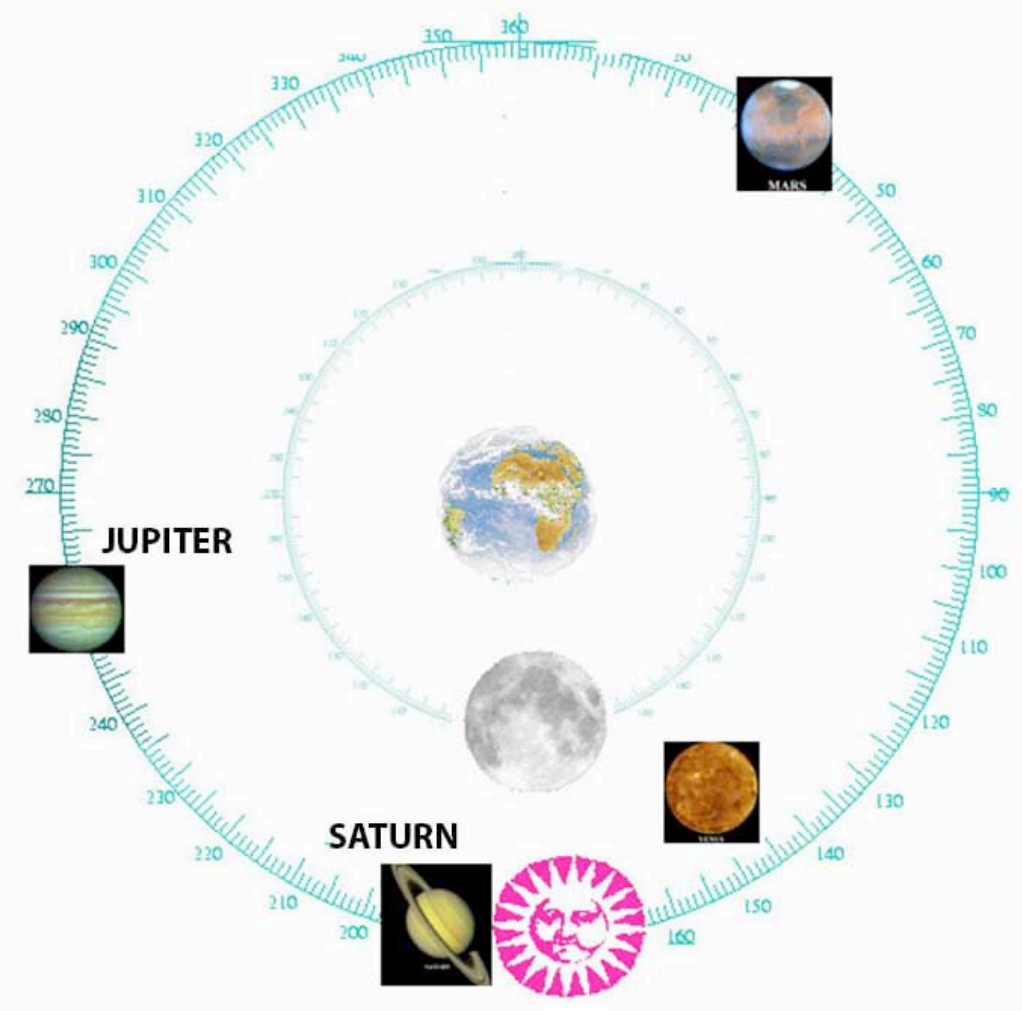

When we used Ptolemy's ( $2^{\text {nd }}$ century AD) values for the mean daily motions of the planets (Toomer, 1984), the corresponding set of positions for 177.18356 days $[=(29.5+793 / 1080 / 24) \times 6]$ earlier, were found to be as in Table 4. 
Table 4. The calculated approximate positions of the outer planets in the first day of the month of Nisan. (See Figure 7).

\begin{tabular}{l|c|c|c}
\hline & $\begin{array}{c}\text { Ptolemy's values for the daily } \\
\text { motion of the Planets. } \\
\text { Degrees/day }\end{array}$ & Mean 177.18356 days earlier & $\begin{array}{c}\text { Position of the planet } \\
\text { within 2 degrees }\end{array}$ \\
\hline Saturn & 0.033489 & $179^{\text {th }}$ degree & $\mathbf{1 8 0}$ \\
\hline Jupiter & 0.083122 & $239^{\text {th }}$ degree & $\mathbf{2 4 0}$ \\
\hline Mars & 0.524059 & $302^{\text {nd }}$ degree & $\mathbf{3 0 0}$ \\
\hline Mean sun & 0.985635 & $1^{\text {st }}$ degree & $\mathbf{0}$ \\
\hline
\end{tabular}

Figure 7. The clear unique order as visualized by the sages for the day of creation. The outer circle represents the daily motion of the mean sun. On the first day of creation the outer planet Saturn was first created and after 4 hours of the rotation of the outer circle Jupiter was creates and so on. The Sun was created on a Wednesday at 6 am and started to circle the Earth. At 10 am Venus was created (See Appendix I) and at $2 \mathrm{pm}$ - Mercury. Finally, close to $6 \mathrm{pm}$, at the end of Wednesday, the moon was created forming a New-Moon since the Sun and the moon have the same celestial longitude. This Figure was created by the author.

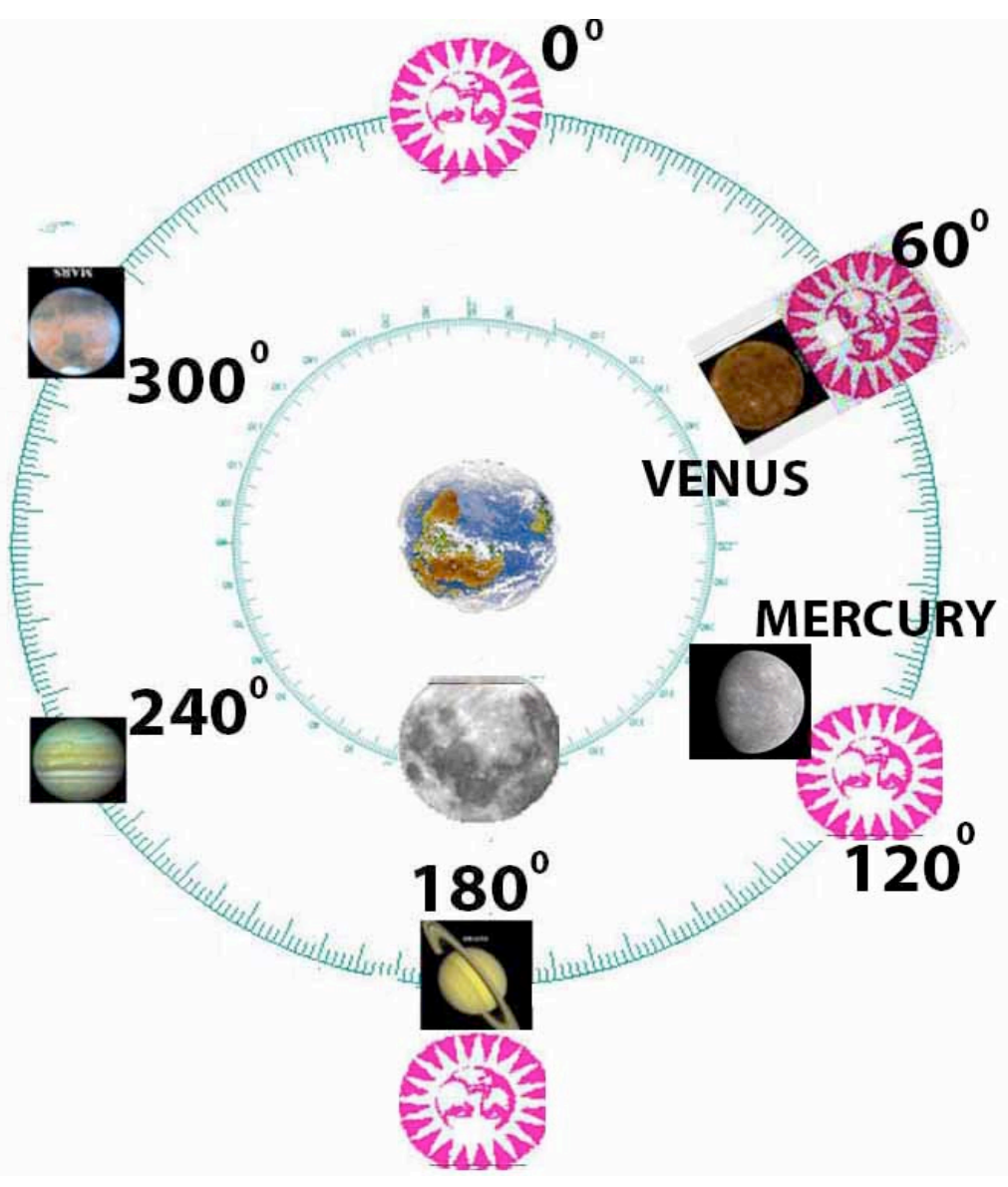

The most important part of the puzzle has been solved: We now have the "big bang" of the Biblical chronology. We now know that we have to go back and look for the year in which we can find such an arrangement of the planets implementing a similar method used by Aryabhatta in the Hindu tradition. In particular, we should look for a new moon occurring at the end of the first day of the spring (See Fig. 7), the day when all stars including the sun and the moon were formed, the weekday of which is Wednesday based on the first chapter of the Old Testament.

We note that the positions of the planets presented for "Adam's new moon" in the first column of al-Khwarizmi's Table are not given with a high precision as the one assigned to the positions of the planets in the days of the building of the Temple. We, therefore, conclude that our calculated mean positions of Saturn, Jupiter, and Mars (being all 
within 2 degrees from the exact longitudes of 180, 240, and 300 degrees), suggest that the approximate mean longitudes are used to indicate the implied fact that all outer planets were believed to have been created at the exact well organized real longitudes, whereas the determination of their exact mean longitudes was not required for the visualization of their creation.

In Appendix 1 we describe additional indications that the Table refers to the creation of the planets at the specific celestial longitudes being exactly 60 degrees apart including the inferior planets, Venus and the moon. In Appendix I we provide the detailed calculation of the position of the inferior planet Venus in day one having the same real celestial longitude as the sun.

As to the moon, the position of the mean moon along with our modified information regarding the lunar apogee and the lunar node, when calculated backwards, are resulting in a unique well determined total eclipse: Based on the celestial longitudes of the lunar node and the lunar apogee it can be shown that exactly 10 months prior to the autumnal new moon in the Table, the lunar node was at the longitude of $179^{\circ}$ (the descending longitude of the moon in its tilted orbit relative to the ecliptic plane) which corresponds to an eclipse when the sun is on the celestial longitude of $1^{0}$. The lunar apogee calculated backwards for the discussed new moon is on the $1^{0}$ longitude causing the eclipse to be a total eclipse. The new moon being an eclipse 10 months and not 6 months prior to the autumnal new moon is either a result of an error made by the sages who changed the original values of both the lunar apogee and lunar node with the same error, or was calculated with the intention to masquerade the modification of the Table from a spring new moon to an autumnal new moon for the creation of the world.

The illustration of the planets in the first day of creation is presented in Figure 8, in which the hours of Wednesday indicate the time in which the corresponding planet was in the Eastern Horizon (Zenith Angle $=90^{\circ}$ ).

Figure 8. The resulting visualization of the planets' orbits and their time of creation in the day Wednesday of the creation of the planets including the sun and the moon. $6 \mathrm{pm}$ for Saturn refers to Tuesday (the beginning of the Jewish Wednesday) and to close to the end of Wednesday in reference to the moon. The background Ptolemaic view of the orbits can be found in http://www.hellenicaworld.com/Greece/Science/en/PtolemyAstronomy.html. The times of creation of the planets as well as their pictures were added by the author.

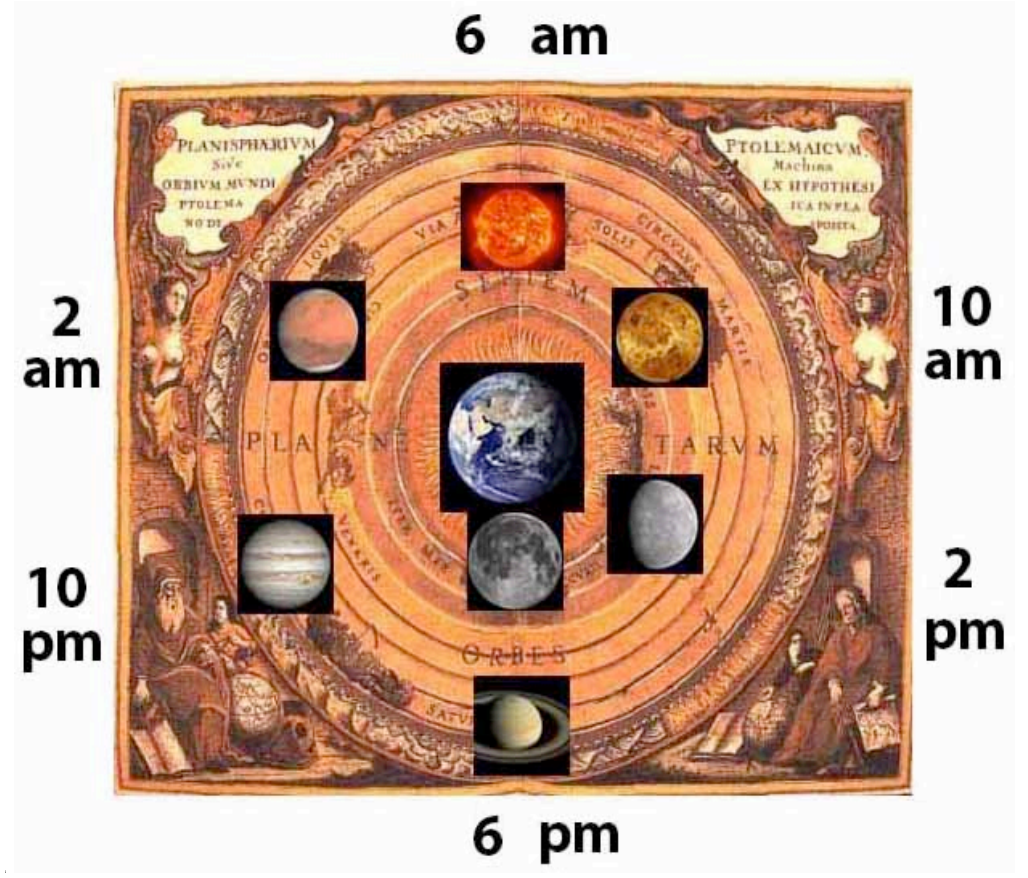

\footnotetext{
CALCULATING THE ORIGINAL YCR BASED ON THE MODIFIED AL-KHWARIZMI'S TABLE Copyright by author(s); $\underline{\text { CC-BY }}$ 
Maimonides devoted two chapters (Maimonides, $12^{\text {th }}$ century A.C.) to describe the methods used by the ancient Jews in the times of the Sanhedrin ( $3^{\text {rd }}$ century B.C. to $3^{\text {rd }}$ century A.C.) to calculate the time difference in any given year between the new moon of Nisan (the first lunar month of the spring) and the beginning of spring. The first of the two chapters outlines the method used by the ancient sages who applied the value of 365.25 ( $\left.\mathrm{Y}_{1}\right)$ days for the length of the tropical year, in addition to the present value of the length of the mean month in the Hebrew calendar, being 29 days, 12 hours, 44 minutes and $31 / 3$ seconds $\left(\mathrm{M}_{\mathrm{H}}\right)$. This length of the month had been known to the Babylonians as early as the $2^{\text {nd }}$ century B.C., and was used by Ptolemy in his Almagest (Toomer, 1984) in the middle of the $2^{\text {nd }}$ century A.C.

In contradiction to the present permanent Hebrew calendar, to be discussed in the next section, in a cycle of 19 years, 235 of the above mentioned mean lunar months, are not equal to 19 years of 365.25 days. The difference after 19 years is close to 87 minutes:

$$
235 \times \mathrm{MH}_{\mathrm{H}}-19 \times \mathrm{Y}_{1}=0.06 \mathrm{~d}=86.944 \mathrm{~m}
$$

Clearly, this difference is increasing by -86.944 after every cycle of 19 years. But other large cycles can be determined to make the difference between integer number of years and a corresponding number of lunar months. Indeed, using the Continued Fraction approach we find that after 1468 year $=536187$ days, the difference between 1468 years and 18157 months is 3.278 minutes:

$$
1468 \times \mathrm{Y}_{1}-18157 \times \mathrm{M}_{\mathrm{H}}=0.00228 \mathrm{~d}=3.278 \mathrm{~m}
$$

The sages who were aware of this large cycle (using a different methodology), used two of such cycles, namely 2936 years, as a time difference between the "Building of the Temple" (second column of data in al-Khwarizmi's Table, see Table 2) and the "First of the Days of Adam". Therefore, the new moon related to the two events took place in practically the same celestial longitude as stated in Table 2. We note that al-Khwarizmi thought that the same celestial longitudes are resulting from an integer number of 19 year cycles since the new permanent Hebrew calendar assumed that after 19 years the celestial longitude of the mean sun remains unchanged. But his interpretation was incorrect because, to begin with, he was also unaware of the fact that the modified New Hebrew calendar he was trying to describe had 2928 years between the two event and $2928 \bmod 19=2$. Furthermore, the values of the coordinates of the mean sun and the mean moon in the 3rd (last) set of data in the Table can be explained only when the length of the year is 365.25 days and not 235 months divided by 19 which is the length of the year in the Hebrew calendar - see the next section. In that respect we would like to emphasize that according to the Jewish permanent calendar in a cycle of 19 years in which the length of the year is exactly 235 months, each celestial longitude of the mean sun has a one to one correspondence to exactly 19 celestial longitudes of the mean moon. The pair cited by al-Khwarizmi at the beginning of the Seleucid era is not an allowed pair if in year 1 they are both in exactly the longitude 176.000 with an accuracy similar to the one assigned to the other planets in the beginning of Solomon's Temple. Instead, when we take the sum of one cycle of 483 years and add 25 years of 365.25 days, we end up with exactly the same celestial longitudes of the sun and the moon as appear in al-Khwarizmi's last column of Table 2. We also remark that it makes little sense to suggest in an astronomical table that two of the major events in the history of the Hebrews start we the same exact 19 year cycles' value when the number of such cycles in 2926 years is 154 . We claim that the table provides a unique significance to the same celestial longitudes of the new moons of the 2 events when they denote a separation of two major cycles of 1468 years each. This cannot be considered as merely a result of a coincidence.

With our conclusion that these large cycles are involved we can proceed and enlighten the methodology used in the determination of the YCR. First, we note that:

$$
2936 \text { years }=1072374 \text { days, constitutes of an integer number of weeks }+2 \text { days. }
$$

In addition, since 2936 years are a multiple of 4, the spring after 2936 years starts at the same time of the day, as in the day of creation.

Consequently, all that the astronomer, advising the Jewish sages, had to do in order to determine the YCR, was to look for a mean new moon that took place on a Friday at 6 PM in Jerusalem (2 days after the creation's weekday of 
Wednesday (See eq. 3)), a day after the beginning of spring within the $9^{\text {th }}$ or the $10^{\text {th }}$ centuries B.C (in which they assumed that Solomon's Temple was built), which would satisfy the above mentioned constraints. Once such a new moon is found, the YCR would be 2936 years earlier in time.

By using Table VI 6 "of the conjunctions of sun and moon" in the Almagest (Toomer, 1984, p. 278), it can be determined that the new moon close to the beginning of spring on a Friday in the year 821 B.C., and, consequently, on a Wednesday in the year 3757 B.C., are the only ones that took place in Jerusalem at 6 PM (within 10 minutes).

The importance of this result is accentuated in three main conclusions:

a. The visualization of the time of the new moon relative to the spring equinox help determining the YCR as well as the year in which Solomon's Temple was built.

b. A major event such as the building of the Temple is believed to have taken place when the sky had the sun, the moon, and the zodiac are at exactly the same relative coordinates.

This last conclusion resembles the basic hypothesis defining the Hindu calendar according to which its future reborn world will start its new cycle when all planets are realigned (See Figure 1).

c. It is our claim that the determination of the year related to the building of Solomon's Temple, used in the biblical chronology, is merely dependent on astronomical calculations. Indeed, it is off by over 140 years from its, later determined, historical accepted value.

\section{CHANGES IN THE DETERMINATION OF THE YCR IN THE HEBREW CALENDAR RESULTING FROM DEVELOPMENTS IN ASTRONOMY}

During the last centuries B.C. and the first Millennium A.C. the astronomical world realized that the length of the tropical year $Y_{1}$ could not be measured accurately by methods like the measurement of the seasonal changes of the levels of the Nile, and it became recognized that the value of $\mathrm{Y}_{1}$ should be reduced. In his Almagest Ptolemy followed Hipparchus and reduced its value by $1 / 300$ of a day making it equal to $365.24667\left(\mathrm{Y}_{\mathrm{A}}\right)$ days. The Almagest was considered among astronomers as the main source for their calculations for several centuries till the middle of the second Millennium. This fact had a very pronounced effect on calendars in general and on the determination of the YCR as did the change of the Hubble constant which was followed by a corresponding effect on our understanding of the age of the Universe.

In his second chapter mentioned above Maimonides (Maimonides, 12 $2^{\text {th }}$ century, ch. 10 [English translation. 1956]) states that other Jewish sages (after the Talmud era ending in the $5^{\text {th }}$ century A.D., during which the only tropical year mentioned in Jewish sources was $\mathrm{Y}_{1}$ ), decided to use a value which was close to the Almagest value for a permanent calendar which relied merely on astronomical calculations. But, in order to facilitate the calculations, they based their determination of the length of the tropical year, $\mathrm{Y}_{\mathrm{H}}$, on a cycle of 19 years in which there are exactly 235 mean lunar months equalling $\mathrm{M}_{\mathrm{H}}$ :

$$
\mathrm{Y}_{\mathrm{H}}=235 \times \mathrm{M}_{\mathrm{H}} / 19=365.2468222 \mathrm{~d} \text {. }
$$

Hence, $\mathrm{Y}_{\mathrm{H}}=\mathrm{Y}_{\mathrm{A}}+13.44$ seconds.

The YCR can no longer be visualized by the unique order described above in Figure 8, and it was only required that the new moon of the day of creation of Adam will be the first new moon of the autumn and that it should be on a Friday, the weekday when as written in Genesis Adam was created. According to Maimonides (Maimonides, $12^{\text {th }}$ century) the new moon 6 months earlier was within 1 day from the beginning of spring. 
The year that was satisfying such new requirements and was as close as possible to the year 3757 B.C., was the year 3760 B.C., 3 years earlier, which is the present year of the creation of Adam in the Jewish calendar a year that starts with "Adam's New Moon" (year 2 of the calendar).

But, the original astronomical table had to be modified accordingly before being presented by al-Khwarizmi. The sages from whom al-Khwarizmi received the astronomical table calculated the new celestial longitudes of the planets including the sun and the moon in two steps. First simply by multiplying the daily motion of the planets by 6 months and adding the results to the original coordinates, making sure that the new moon would represent the celestial longitudes of the autumn. Then by realizing that in 3 years the celestial longitude of Jupiter varied from being in front of Saturn in 3757 B.C. to a celestial longitude behind Saturn in the year 3760 B.C., and since in the new era starting with Adam's new moon, there was no more a unique arrangement of the planetary celestial longitudes, they just interchanged the original values between Saturn and Jupiter. Indeed, even though the length of the tropical year of the Almagest is different from the year used in the original Table, Figure 9 shows a general agreement for the real celestial longitudes calculated by use of the Almagest for the year 3757 B.C. (upper left of the Figure) whereas there is no resemblance between al-Khwarizmi's modified Table and the Almagest results for the year 3760 B.C. (upper right of the figure).

Figure 9. The celestial longitudes of the planet as calculated using the Almagest Ephemeris Calculator in R. H. van Gent's http://www.staff.science.uu.nl/ gent0113/astro/almagestephemeris.htm

On the left hand side on the first row, we present the almagest calculated celestial longitude for the date: Wednesday, April 2, $3760 \mathrm{BCE}$, which is the date corresponding to the Permanent Jewish Calendar. The Celestial coordinates are compared with our calculations derived from alKhwarizmi's manuscript (bottom left).

On the right hand side on the first row, the almagest celestial coordinates are calculated for the date: Wednesday, March 29, 3757 BCE, showing a much better agreement with our calculation (bottom right). The figure was prepared by the author.
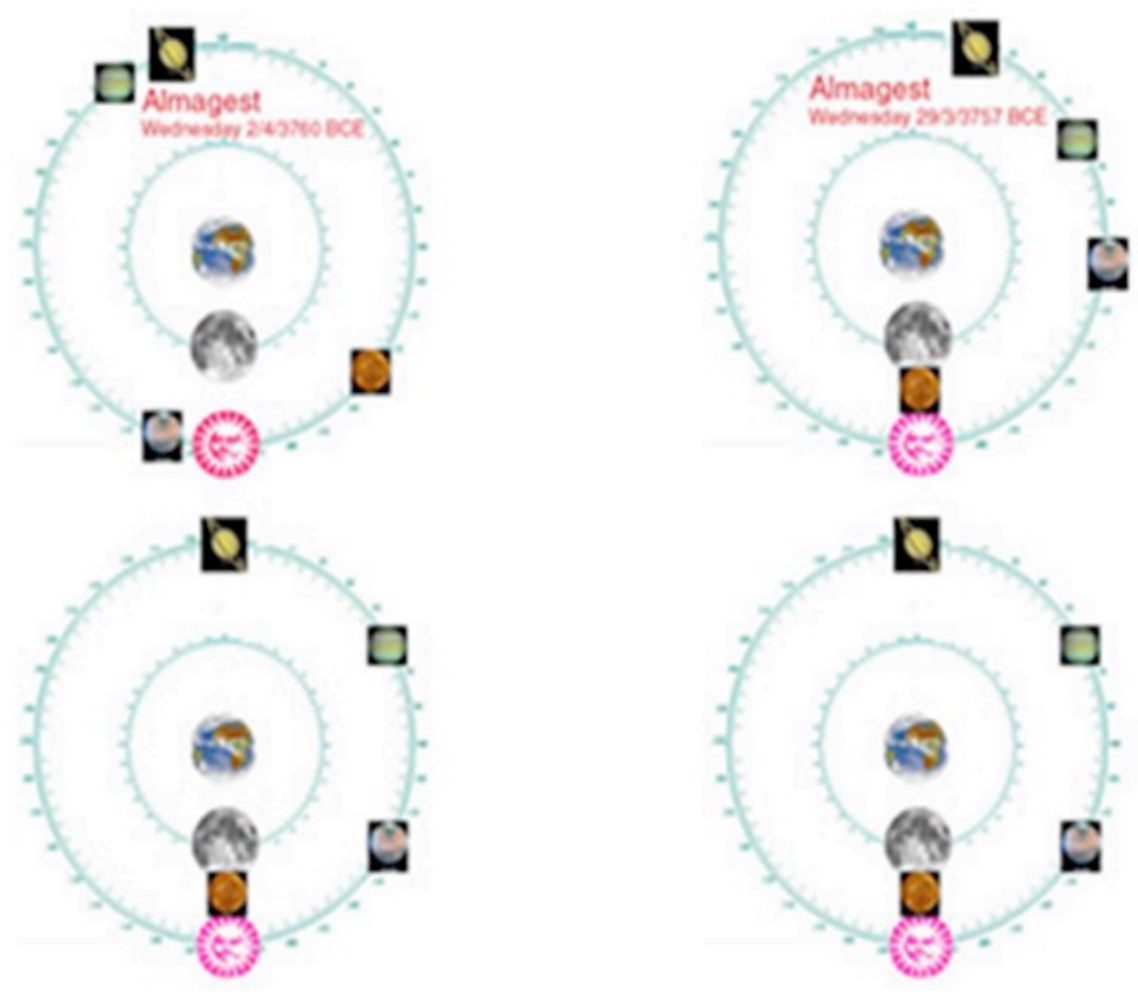


\section{CHANGES IN THE DETERMINATIONS OF THE YCR FROM THE HEBREW TO THE GREEK ORTHODOX CHRISTIAN CHURCH (OR VICE VERSA) RESULTING FROM DEVELOPMENTS IN ASTRONOMY}

From the last few centuries B.C. major cultures in the Middle East used for the length of the mean lunar month a value of 29.5 days $+44 \mathrm{~m}\left(\mathrm{M}_{\mathrm{A}}\right)$. Such a value has been in use in the Islamic world ever since the $7^{\text {th }}$ century A.C. as well as by the Jews during the last centuries B.C. In addition, this length of the month was used in the Hindu culture to within 0.3 of a second as emphasized in Figure 1 .

It is claimed by us that the Septuagint version which was the result of the translation of the contemporary Old Testament by seventy sages from the 12 Tribes of Israel into the Greek language in the $3^{\text {rd }}$ century B.C., has a chronology which had been based on astronomical calculations using the length for the tropical year $\mathrm{Y}_{1}$ and $\mathrm{M}_{\mathrm{A}}$ for the length of the month.

With our conjecture that the main visualization resulting from our modified Table of al-Khwarizmi also applied to the chronology of the Septuagint version, the first new moon took place at the same day as the beginning of spring.

Therefore, the YCR cannot be the same as in the Masoretic version since going back in time with a different length of the month leads to a different YCR in which the above mentioned constraints apply. In order to reach a different YCR dissimilar ages of the Patriarchs can contribute in reaching that goal.

When we used the continued fraction approach for the relative values of $Y_{1}$ and $M_{A}$, we found that after a grand cycle of 426 years a new moon would occur in practically the same celestial longitude as in the time of creation:

$$
426 \times \mathrm{Y}_{1}-5269 \times \mathrm{M}_{\mathrm{A}}=4 \text { minutes. }
$$

We, therefore, suggest that the basic large cycle in the Septuagint version is thus 426. According to al-Khwarizmi Table, the building of Solomon's Temple is such an important event in the history of the Hebrews in the Old Testament that it is accompanied with the same new moon at the end of the first day of the spring as in the day of creation. Indeed 10 cycles of 426 years after the day of creation, namely, 4260 years, separate between the two events in the biblical chronology of the Septuagint version (the Vaticanus manuscript), as detailed in Table 5.

Table 5. Number of years that passed between the birth of Adam and the building of the First Temple, according to the Septuagint

\begin{tabular}{|l|c|c|c|c|}
\hline & Cycle Number & $\begin{array}{c}\text { Sum of } \\
\text { Astronomical } \\
\text { Cycles }\end{array}$ & $\begin{array}{c}\text { Comparison between the start of } \\
\text { the Large Astronomical Cycles } \\
\text { and the Event According to the } \\
\text { Biblical Chronology }\end{array}$ & $\begin{array}{c}\text { \# of Year starting } \\
\text { from the Creation } \\
\text { of the World }\end{array}$ \\
\hline First Spring of Creation & $\mathbf{0}$ & $\mathbf{1}$ & $3315^{*}$ \\
\hline Birth of Abraham & & & & 3380 \\
\hline Adam Enters the Land of Israel & & & & $3820^{* * *}$ \\
\hline Exodus & 10 cycles & $+426 \times 10$ & & \\
\hline Year of Building the $1^{\text {st }}$ Temple & & $\mathbf{4 2 6 0}$ & & \\
\hline
\end{tabular}

*2144 (Table 2) + 100 (Shem) + 135 (Arphaxad) +130 (Cainan) + 130 (Salah) + 134 (Eber) + 130 (Peleg) + $132($ Reu $)+130($ Serug $)+79($ Nahor $)$ $+70($ Terah begot Abraham $)=3314$ years.

** According to the Septuagint, 505 years passed between the birth of Abraham and the Exodus.

*** Number of years between Exodus and the building of Solomon's Temple - In https://www.biblica.com/bible/niv/1-kings/6/, we find: Kings I 6:1 In the four hundred and eightieth ${ }^{[a]}$ year after the Israelites came out of Egypt, in the fourth year of Solomon's reign over Israel, in the month of Ziv, the second month, he began to build the temple of the lord. ${ }^{[a]} \underline{1 \text { Kings } 6: 1}$ Hebrew; but Septuagint four hundred and fortieth.

The fact that there are 4260 years separating the YCR and the Temple's first year has been known in the past to various researchers. For example, J. Hughes states specifically that "the first year of the exodus is 3820 AM, the temple's first 
year is $4260 \mathrm{AM}$, and", he adds, "the first year after the destruction of the temple is 4690 AM" (Hughes, 2009, p. 239). However, the 4260 years has remained without an explanation, except by us (Cohen, 2009 [in Hebrew]). However, as we claim, al-Khwarizmi's manuscript provides the answer: Astronomy had been used by ancient cultures to look for celestial signs in order to determine the years of major events (See Conclusions) thus providing the necessary tools to the ancient's understanding of the history of mankind.

We note that there is a disagreement between different ancient scholars regarding the exact YCR of the Septuagint chronology - see Table 6

Table 6. Various years of creation as assumed by ancient sages (See, for example, Gibbon, 1906).

\begin{tabular}{|c|c|}
\hline The YCR according to the Septuagint & Source \\
\hline 5529 B.C. & Theophilus of Antioch \\
\hline 5509 B.C. $(=-5508)$ & Greek or Byzantine Era \\
\hline 5501 B.C. & Africanus and Hippolytus of Thebes \\
\hline 5501 B.C. & Annianus \\
\hline 5501 B.C. & Syncellus and Theophanes \\
\hline 5494 B.C. & Dionysius Exiguus \\
\hline 5199 B.C. & Eusebius \\
\hline
\end{tabular}

It can be seen that Eusebius' interpretation for the Septuagint's YCR, 5200 B.C., leads to the nearest historical year of the building of Solomon's Temple. $5200-4260=940$ against the year 962 B.C., which is the year set by the historians for this event.

The determination of the YCRs in the table will be discussed separately.

\section{CONCLUSIONS}

Astronomical data and astronomical calculations had played an essential role in ancient cultures. The rare table presented by al-Khwarizmi is an exceptional document revealing such a role in the Middle Eastern ancient cultures in addition to the central role in the Hinduism in which the world eras are known to have been related to astronomical data. It is our understanding that in contradiction to the distinction in the last Millennium between religion and astrological beliefs, practically all ancient cultures did not distinguish between religion and astronomy. The understanding of the role of astronomy to the ancient cultures is an important information that all scholars and students of astronomy must share. The tools required to comprehend the traditions based on astronomy can only be appreciated by scholars and students who have a basic apprehension of the science of astronomy.

Astronomy was used by the ancient cultures and religions for dating past major events in their history for which no other document had existed (this is in a complete dissimilarity to astrology which is believed by many to predict the future or to explain present characters). In fact the inability to date the past was not only limited to the creation of the world. All the main cultural events described in the bible, can be shown to have been linked to large astronomical cycles in the Masoretic version of the Bible. The continued fraction approach shows us that the large cycle of 1468 years discussed above is composed of 3 smaller cycles of 483, 483 and 502 years:

$$
\begin{aligned}
483 \times \mathrm{Y}_{1} & =5974 \times \mathrm{MH}_{\mathrm{H}}-27.89 \mathrm{~m} \text {, and } \\
502 \times \mathrm{Y}_{1} & =6209 \times \mathrm{MH}_{\mathrm{H}}+59.06 \mathrm{~m}
\end{aligned}
$$

Consequently, after each such cycle of 483 or 502 years the new moon and the beginning of spring will be within less than an hour as they were in the day of creation. As can be seen in Appendix II the major events, namely, the birth of Abraham - the father of the Hebrews, Exodus, the building of Solomon's Temple, and the building of the second temple as interpreted from the text were all in general agreement with such cycles. The agreement with astronomical cycles were considered as the most important clue to the dating of the events. Even though the historians came out 
with dates over 140 and 160 years apart from the corresponding biblical dating of the building of each of the two temples, the biblical chronology of the Masoretic text as interpreted in the $2^{\text {nd }}$ century A.C. by Joseph ben Halafta, has remained the official biblical chronology of the Masoretic version in the Jewish religion.

Finally, we remark that two different chronologies cannot be regarded by the Greek Orthodox Church or by the Catholic Church and the Jews to be both sacred. In the Jewish religion, for example, the Masoretic text is considered sacred whereas the Septuagint chronology is regarded by the Jewish orthodoxy as a blasphemy. We, therefore, feel it necessary to emphasize that no attempt is made by us to imply what version is the original version the chronology of which served as the basic data for the modifications made in the chronology in general and in the Patriarchs' ages in particular when they begot their next generations (Table 1). In fact, our approach is purely scientific, an approach which should also include an assumption that both versions may not be the original version, as we mentioned in Section 1. The book of Jubilees is assumed to have been written even before the original Septuagint version and it contains a chronology which is different from the Septuagint version and only partially resembles the Masoretic chronology.

But, as we showed above, one way or another, there is no doubt that astronomy played a critical part it the formation of our cultures and in our understanding of the progress of science in the ancient world. The study of the development of calendars from the ancient world till the present times is thus a major tool for teaching the history of science in general and the history of astronomy in particular. Such a teaching method provides a profound understanding of the fact that the progress of science has been a constant instrument that could not have been disregarded by cultural or religious restrictions for an unlimited amount of time. Science has proved to find its ways to be triumphal over any restrictions imposed on its brave creators of science and astronomy throughout the history.

The teaching of the history of science allows the students to amend their a-priori beliefs that science has been always in a direct confrontation with religion. However, in fact, they can learn that the ancient world, in general, had known better to adopt new cultural-religious rules with the progress of astronomy and science than the present cultural regulations seemingly imposed, every so often, by religious dogmas.

\section{AUTHOR BIOGRAPHY}

Dr. Cohen is the Professor in the Institute of Earth Sciences at the Hebrew University of Jerusalem and Chairman of the Jerusalem Society of Science, Computers and Software Engineering. In addition to his considerable experience teaching Earth and space sciences, he was among the founding scientists for introducing remote sensing research in Israel, including laser radar systems and satellite systems. E-Mail: cohariel@gmail.com

\section{REFERENCES}

al-Khwarizmi, M. ibn M. (820 A.C).: Fi istikhraj ta'rikh al-yahud ("letter on the extraction of the Jewish calendar"), folios 115v$117 r$ of MS. Bankipore Arabic 2468 in the Khuda Bakhsh Oriental Public Library, Patna, India - See also Kennedy below.

Burgess, E. (1860). Surya-Siddhnta, a text-book of Hindu astronomy. New Haven, The American Oriental Society.

Cohen, A. (2004). Long term astronomical cycles calculated by astronomers during the first millennium AD and their impact on the Jewish chronology, Judea and Samaria Res. Studies, 13 (Edit. J. Eshel), 331-343 (in Hebrew with an English Abstract).

Cohen, A. (2009). Journey to the history of astronomy: The decoding of the biblical year of creation (In Hebrew). Jerusalem, Ido's Book Publishing.

Frank, E. (1956). Talmudic and Rabbinical chronology. New York, Philipp Feldheim, Inc., Publishers.

Gibbon, E. (1906). The History of the Decline and Fall of the Roman Empire. New York, Fred de Fau and Co. Ch 3. World Eras - (page 25).

Hughes, J. (2009). Secrets of the times: Myth and history in biblical chronology. Sheffield Academic Press.

Kennedy, E. S. (1964). al-Khwarizmi on the Jewish Calendar. Scripta Mathematica, 27, 55-59.

Maimonides, M. (12 ${ }^{\text {th }}$ century A.C.). Laws of sanctification of the new year (LSNY) Chaps. 9 and 10, English translation by Gantz, Neugebauer, et al. (1956).

Scaliger, J. (1583). De Emendatione Temporum.

Toomer, G. J. (1984). Ptolemy's Almagest. London, Duckworth.

van Gent, R. H., (2007) Almagest Ephemeris calculator. http://www.staff.science.uu.nl/ gent0113/astro/almagestephemeris.htm.

Copyright by author(s); $\underline{\mathrm{CC}-\mathrm{BY}}$ 


\section{APPENDIX I}

\section{The Position Of The "Inferior" Planet Venus In The Day Of Creation Of The Stars.}

Table 7. The number of degrees which Venus completes in 177.55 days along the epicycle.

\begin{tabular}{|c|c|c|}
\hline $\begin{array}{c}\text { Ptolemy's value for the daily motion } \\
\text { of Venus on the epicycle }\end{array}$ & Degrees/day & $\begin{array}{c}\text { Total motion on the epicycle in } 6 \\
\text { months or 177.5521 days }\end{array}$ \\
\hline
\end{tabular}

Table 2 suggests that the planet Venus was at the celestial longitude of the $145^{\text {th }}$ degree when the mean sun was at the $176^{\text {th }}$ degree.

From the celestial coordinate of the real moon on the day of creation being at the same longitude as the real sun, we can assume that the inferior planet Venus, when believed to have been created, was also within 2 degrees from the vernal equinox. Therefore, the position assigned in Table 2 to Venus six months after the spring equinox can only represent its true longitude since the anomaly varies by 109.5 degrees in 177.55 days (Table 7) and not $144+/-2$ degrees (Table 2).

In order to find the longitude of Venus relative to the mean sun after advancing $109.5^{0}$ along the epicycle, we have assumed, for the first approximation, that Figure 10 was used by the ancients for the calculation of the real longitude neglecting the effect of the position of the eccenter. With the distance of the mean sun, R, being $40 \%$ larger than the radius of the epicycle, $r$, and for an angle of $\mathrm{VCT}=109.5^{\circ}$, we get VTC to be 28.5 degrees (Fig 10). This approximate result is within 2.5 degrees from the difference between the position of the mean sun and the position assigned to Venus in Table 2.

Figure 10. The simplified geometric model for epicyclical motion of the inferior planet Venus denoted by $\mathrm{V}$. T is the earth and $\mathrm{C}$ is the mean sun. $\mathrm{R} / \mathrm{r}=1.4$. This model was created by the author for discussing the celestial longitude assigned to Venus in the day of creation: $\mathrm{VCT}=144+/-2$.

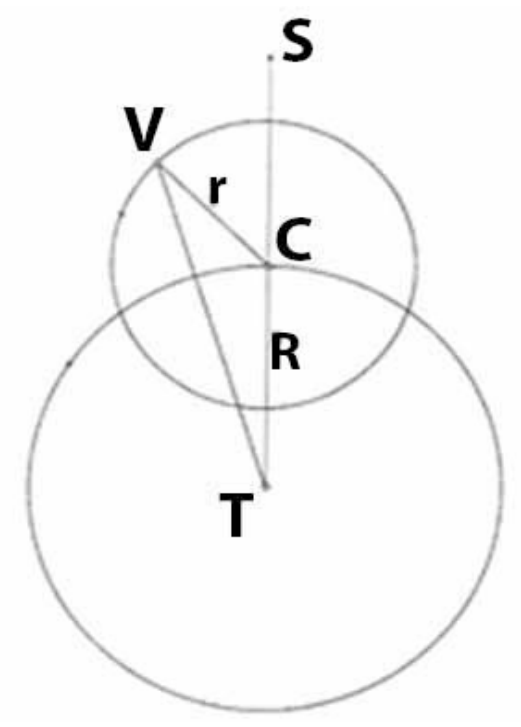

However, we note that the use of the exact Ptolemy's equations for the determination of the position of the inferior planet during the mean new moon of Adam, leads to an angle of $30.2^{0}$ from the longitude of the new moon, a result which is within less than $1^{0}$ from the listed difference in Table 2 between the real planet and the mean sun. 


\section{APPENDIX II}

Comparison between the major events in Jewish History in the Talmudic chronology (as presented by Frank, 1956). The Table is based on our Table in Cohen, 2004.

\begin{tabular}{|c|c|c|c|c|c|}
\hline Year B.C. & $\begin{array}{c}\text { Number of } \\
\text { months from the } \\
\text { day of creation }\end{array}$ & $\begin{array}{l}\text { Serial Number } \\
\text { of Long Cycle }\end{array}$ & $\begin{array}{c}\text { Number of years after } \\
\text { creation based on the } \\
\text { Astronomical large } \\
\text { cycles }\end{array}$ & $\begin{array}{l}\text { Number of years after } \\
\text { creation according to } \\
\text { the Masoretic version }\end{array}$ & $\begin{array}{c}\text { Event In the } \\
\text { biblical } \\
\text { Chronology }\end{array}$ \\
\hline \multirow[t]{5}{*}{3757} & & 0 & 0 & 0 & $\begin{array}{l}\text { Creation of } \\
\text { Man }\end{array}$ \\
\hline & & & & $1656+$ & \\
\hline & & & & 1656 & The Deluge \\
\hline & & & & $292+$ & \\
\hline & & & $\begin{array}{ll}+483 & +502 \\
+483 & +483\end{array}$ & & \\
\hline \multirow[t]{3}{*}{1806} & 24131 & 4 & 1951 & $\begin{array}{l}1948 \\
100+\end{array}$ & $\begin{array}{l}\text { Birth of } \\
\text { Abraham }\end{array}$ \\
\hline & & & & 2048 & Birth of Isaac \\
\hline & & & +502 & $400+$ & \\
\hline \multirow[t]{3}{*}{1304} & 30340 & 5 & 2453 & $2448 \quad 2453^{*}$ & Exodus \\
\hline & & & & $40+$ & \\
\hline & & & +483 & $\begin{array}{l}2488 \\
440+\end{array}$ & $\begin{array}{l}\text { Entering the } \\
\text { Land of } \\
\text { Canaan }\end{array}$ \\
\hline \multirow[t]{4}{*}{821} & 36314 & 6 & 2936 & $2928 \quad 2933^{*}$ & First Temple \\
\hline & & & & $410+$ & $\begin{array}{l}\text { Years of the } \\
\text { Temple }\end{array}$ \\
\hline & & & & 3338 & $\begin{array}{l}\text { Destruction of } \\
\text { the Temple }\end{array}$ \\
\hline & & & +483 & $70+$ & $\begin{array}{l}\text { Babylonian } \\
\text { Exile }\end{array}$ \\
\hline \multirow[t]{2}{*}{338} & 42288 & 7 & 3419 & $\begin{array}{c}3408 \quad 3413^{*} \\
+40\end{array}$ & $\begin{array}{l}\text { Second } \\
\text { Temple }\end{array}$ \\
\hline & & & & 3448 & $\begin{array}{l}\text { Start of the } \\
\text { Seleucid Era }\end{array}$ \\
\hline
\end{tabular}

* According to the Catholic Church as emphasized by Joseph Scaliger in De Emendatione Temporum (1583), 505 years passed from the birth of Abraham to Exodus and therefore there is an exact fit between the calculated large cycles in the table and the year of Exodus being 2453 years. 